\title{
FUNCTIONAL LINK NETWORK WITH GENETIC ALGORITHM FOR EVOKED POTENTIALS
}

\author{
Bor-Shyh Lin, Bor-Shing Lin, Jen-Chien Chien, FoK-Ching ChOng \\ Department of Electrical Engineering, National Taiwan University, Taipei, Taiwan
}

\begin{abstract}
In this study, a functional link network with genetic algorithm is employed for extracting evoked potentials. Evoked potentials are time-varying signals typically buried in relatively large noises of electroencephalogram. Recently, to extract evoked potentials more effectively from noises, adaptive filtering techniques are widely employed for evoked potentials. In general, least-mean-square algorithm is used to adapt filter weights. However, it is well-known that the selection of step-sizes is a trade-off on the convergence rate and steady state performance. In practice, an inappropriate stepsize always causes deficiency. Therefore, the proposed method employs genetic algorithm to improve this issue. Genetic algorithm is basically a kind of evolutionary strategies. The step-size candidates are regularly generated and evaluated, and the fittest candidate is selected for subsequent adaptation. Results show that the proposed method is insensitive to the selection of step sizes and reference inputs. It is applicable for extracting EP in different noise levels.
\end{abstract}

Biomed Eng Appl Basis Comm, 2005(August); 17: 193-200.

\section{INTRODUCTION}

Evoked potentials (EP) are electrical responses of the brain caused by external events, such as sounds, lights or electrical stimuli. The changes of latencies and amplitudes of evoked potentials from stimuli provide important information for various neurological disorders. However, evoked potentials are usually heavily buried in ongoing electroencephalogram (EEG). Even their signal to noise ratio (SNR) is less than $-5 \mathrm{~dB}$ to $-20 \mathrm{~dB}$. [1]. In general, ensemble averaging method is used to extract evoked potentials from measured electrical activities of the brain. It is well-known that the ensemble averaging method requires a lot of trials to extract evoked potentials and is easy to smooth out any information under the variation of evoked potentials.

Received: Dec. 15, 2004; Accepted: July 5, 2005

Correspondence: Fok-Ching Chong, Professor

Department of Electrical Engineering, National Taiwan

University, Taipei, Taiwan

E-mail: fcchong93@hotmail.com
Recently, many approaches were investigated to extract evoked potentials. One of these is adaptive filtering techniques [2]-[10]. This technique is simple to design and is different from Wiener filter and Kalman filter on that it need not priori statistic knowledge of interesting signals and noises [2]. Adaptive filtering technique for extracting evoked potentials was first implemented by Thakor in 1987 [3]. For adaptive filtering techniques, providing a suitable reference signal is important. However, in practice, it is difficult for extracting evoked potentials because the measurement of correlated EEG noises is hard. Therefore, several structures with different sources of reference signals were proposed [4]-[8]. Vaz and Thakor proposed the use of a finite number of sine and cosine waves as reference signals to estimate models of evoked potentials in time domain [4]. Laguna et al used a unit impulse sequence synchronized with the beginning of each recurrence as reference signals [5]. Madhavan employed adaptive line enhancement (ALE) which uses the delay version of desired signals as reference signals, to extract evoked potentials [6]. Since the structure of adaptive line enhancement is effective for signal enhancement 
of single channel, it can solve the issue of reference signals for evoked potentials. Svensson employed an exponential averager as a prefilter which can enhance SNR of both desired and reference signals obtained from realistic evoked potentials [7]. Their results presented that the performance of adaptive line enhancement can be significantly improved by using exponential averagers.

In general, least-mean-square algorithm (LMS) is widely used for adapting weights in adaptive filters. This is simple to implement and does not need to measure the pertinent correlation functions [2]. However, it is well-known that its performance is sensitive to the eigenvalue spread of the autocorrelation matrix of reference signals and step-sizes. It is difficult to set a suitable step-size because properties of observed evoked potentials vary from subject to subject in clinical environment.

In this study, genetic algorithm (GA) [11]-[15] was employed to solve the issue of step-sizes. A functional link network (FLN) with an exponential averager was used as the major filter for evoked potentials. The structure of functional link network was proposed by Pao in 1989 [16]. It has a similar structure to the three-layer multilayer perceptron, except functional expansion units are used in the hidden layer. It can approximate any continuous nonlinear functions if they are fed with adequate functional expansion units in the hidden layer [17]. The genetic algorithm was used to adapt weights. The genetic algorithm provides a parallel search technique which uses the principles of genetics and evolution to perform a directed random search from a population of controllers to select the best to implement at a specific sampling time period. Therefore, it is applicable for solving eigenvalue disparity and local minimum issues via its property of global optimization in control and signal processing area. The remainder of this paper is organized as follows. Section II illustrates the application of an adaptive line enhancement for extracting evoked potentials and the basic scheme of functional link networks. And the implementation of the genetic algorithm is introduced. Results and discussion are presented in Section III. In section IV, conclusions are drawn.

\section{METHOD}

\section{A. Adaptive Line Enhancement for Extracting Evoked Potentials}

The basic scheme of an adaptive line enhancement for extracting evoked potentials is shown in Fig. 1 [7]. It contains two parts: one is the exponential averager, and the other is the major filter.
The exponential averager is used to enhance the SNR of measured evoked potentials before adaptive filtering. Under the assumption of non-stationary evoked potentials, the exponential averager has the ability of rapidly tracking of changes in the evoked potentials [7]. The exponential averager can be treated as an ensemble according to

$$
d(i ; n)=\gamma d(i-1 ; n)+(1-\gamma) E P(i ; n),
$$

where $d(i ; n)$ and $E P(i ; n)$ respectively denote the ith trials of desired inputs in adaptive line enhancement and measured evoked potentials at iteration n. Here, $\gamma,(0<\gamma<1)$ is a forgetting factor. This parameter $\gamma$ is set to 0.8 in this study. Therefore, the variation of measured evoked potentials can be reflected in the desired input after less than 5 trials.

The adaptive line enhancement derived by Widrow et al is used to detect a period-like signal buried in a board-band noise background [2]. It contains two inputs: the desired input and the reference input. In adaptive line enhancement, the reference signal $r(i ; n)$ is highly related to the signal of interest but not necessary same as it. In general, the delayed version of the desired input is used as the reference signal. Consider the following signal model,

$$
d(i ; n)=s(n)+v(i ; n)
$$

where $s(n)$ and $v(i ; n)$ respectively denote the evoked potential of interest and additive uncorrelated noises of $i^{\text {th }}$ trial at iteration $n$. Therefore, the reference signal can be expressed by

$$
r(i ; n)=d(i ; n-\Delta)
$$

Here, the delay $\Delta$ is named the prediction depth of adaptive signal enhancement. The prediction depth is selected to be capable to remove the correlation of the noises in desired and reference signal. Therefore, in practice, we choose the value which approaches the stimulus cycle as the prediction depth. The adaptive line enhancement can be treated as a noise-free model, i.e., the estimate $\hat{s}(n)$ of $s(n)$ can be obtained by the output $y(n)$ of the major filter. In this study, a functional link network and an adaptive line enhancement were used as the major filters.

\section{B. Adaptive Line Enhancement with Normalized Least-Mean-Square Algorithm}

For a M-tap adaptive line enhancement, its output can be expressed by

$$
y(n)=\mathbf{r}(i ; n)^{T} \mathbf{w}(n)=\hat{s}(n),
$$


where $\mathbf{w}(n)=\left[w_{1}(n), w_{2}(n), \ldots w_{M}(n)\right]^{T}$ denotes the weight vector in adaptive line enhancement. Here, $\mathbf{r}(i ; n)=[r(i ; n), r(i ; n-1), \ldots, r(i ; n-M+1)] \quad$ is the vector of reference signals. The weight vector then can be adapted by normalized least mean square algorithm (NLMS). The criterion $\xi$ for adapting weight in this algorithm is defined as the mean square prior estimation error $e(i ; n)$ between desired signals and filtered outputs.

$$
\begin{aligned}
& \xi=\frac{1}{2} \sum_{i}[e(i ; n)]^{2} \\
& e(i ; n)=y(n)-d(i ; n)
\end{aligned}
$$

In order to minimize this criterion $\xi$, gradient descent technique is employed to adapt the filter weights. From equation (4), the gradient of this criterion is given by

$$
\nabla_{\mathbf{w}}(t) \equiv \frac{\partial \xi}{\partial \mathbf{w}(n)}=\mathbf{r}(i ; n)\left[\mathbf{r}(i ; n)^{T} \mathbf{w}(n)-d(i ; n)\right]=\mathbf{r}(i ; n) e(i ; n)
$$

Then the adaptation formula in NLMS can be expressed by

$$
\mathbf{w}(n+1)=\mathbf{w}(n)-\frac{\mu}{\left(\alpha+\mathbf{r}(i ; n)^{T} \mathbf{r}(i ; n)\right)} \mathbf{r}(i ; n) e(i ; n)
$$

Here $\alpha$ is a small constant and $\mu$ is the step-size for NLMS algorithm. The step-size is necessary to obey the sufficient condition $0<\mu<2$.

\section{Functional Link Networks}

The basic idea behind a functional link network is the use of functional links. These functional links generate nonlinear transformations of the original input space before they are fed to the network which constructs the output layer. This basic scheme of FLN is shown in Fig. 2. Structurally a FLN can be broken down into two mappings. The first mapping has input nodes as its inputs and the functional expansion units as outputs. The weights between input nodes and functional expansion units are randomly set. This can impose a topological structure on the input space [16].

Therefore, this mapping is fixed and nonlinear. The second mapping uses the outputs of functional expansion units as its inputs, and its output is a linear sum of functional expansion units. The second mapping is adaptive. Here, sigmoidal functions sigm $(\cdot)$ is employed as the basis function of the functional expansion units in FLN [16] and it is given by

$$
\operatorname{sigm}(x)=\frac{1}{1+e^{-\beta x}},
$$

where, the parameter $\beta$ decides the slope of sigmoidal functions.

For a FLN with $N_{0}$ input nodes and $N_{1}$ functional expansion units, its output can be calculated by

$$
\begin{gathered}
y(n)=\sum_{j=1}^{N_{1}} w_{j 1}^{1} \delta_{j}(n), \\
\delta_{j}(n)=\operatorname{sigm}\left(\sum_{i=1}^{N_{0}} w_{i j}^{0} r(i ; n-i)\right),
\end{gathered}
$$

where $w_{j 1}^{1}$ and $w_{i j}^{0}$ are respectively the weights connecting $j$-th functional expansion unit with the output node and that connecting $i$-th input node with $j$ th functional expansion unit. Here, $w^{0}$ is randomly set. $\delta_{j}(n)$ denotes $j$-th functional expansion unit. The weighted vector $\mathbf{w}^{\mathbf{1}}(n)=\left[w_{11}^{1}(n), w_{21}^{1}(n), \ldots, w_{N_{1} 1}^{1}(n)\right]^{T}$ can be also adapted by using NLMS, i.e.

$$
\mathbf{w}^{\mathbf{1}}(n+1)=\mathbf{w}^{\mathbf{1}}(n)-\frac{\mu}{\left(\alpha+\boldsymbol{\delta}^{T}(n) \boldsymbol{\delta}(n)\right)} \boldsymbol{\delta}(n)(y(n)-d(i ; n)),
$$

where $\delta(n)=\left[\delta_{1}(n), \delta_{2}(n), \ldots, \delta_{N_{1}}(n)\right]^{T}$ is the vector of functional expansion units.

\section{Genetic Algorithm for Functional Link Networks}

The genetic algorithm in this study is developed from normalization NLMS algorithm. It differs from NLMS algorithm in that the generation and evaluation of its step-size are invoked regularly every period of T. The fittest one that provides the optimal performance over an evaluation period is kept and used for the next stage. The basic scheme of genetic algorithm is illustrated in Fig. 3. It mainly consists of three parts: population of filters, fitness evaluation, and the selection and reproduction of step-sizes.

Before population of filters, the reproduction of step-sizes is first occurred at the beginning of the kth interval of evolution. Let $\mu_{k-1, o p t}$ be the optimal stepsize in the $(k-1)$ th interval of evolution. Several offspring of the step-size $\mu_{k-1, o p t}$ are generated by randomly increasing and decreasing $\mu_{k-1, o p t}$. Here, we use random perturbation function [13] as the generating function described as

$$
\left\{\begin{array}{l}
\mu_{k, 1}=\mu_{k-1, o p t} \\
\mu_{k, 2 j}=\left(1-\rho_{2 j}\right) A^{-j} \mu_{k-1, o p t}+\rho_{2 j} \mu_{k-1, o p t} \quad, 1 \leq j \leq \frac{R-1}{2} \\
\mu_{k, 2 j+1}=\rho_{2 j+1} A^{j} \mu_{k-1, o p t}+\left(1-\rho_{2 j+1}\right) \mu_{k-1, o p t}
\end{array}\right.
$$

where $R$ is the number of offspring, $A$ is a positive integer related to the perturbation of new generated step-sizes, and $\rho_{i}, 1<i \leq R$, are random numbers distributed over $[0,1]$. These step-sizes also must fit the convergent condition $0<\mu_{k, i} \leq 2$.

For population of filters, several new filters were formed by using the generated step-sizes. 


$$
\begin{aligned}
e_{k, j}(i ; n) & =y_{k, j}(n)-d(i ; n) \\
\mathbf{w}^{\mathbf{1}}{ }_{k, j}(n+1) & =\mathbf{w}^{\mathbf{1}}{ }_{k, j}(n)-\frac{\mu_{k, j}}{\left(\alpha+\boldsymbol{\delta}^{T}(n) \boldsymbol{\delta}(n)\right)} \boldsymbol{\delta}(n) e_{k, j}(i ; n)
\end{aligned}
$$

where $y_{k, j}(n), \mathbf{w}_{k, j}^{1}(n)$ and $e_{k, j}(i ; n)$ respectively denote the filtered output, weight vector and priori estimation error at iteration $n$.

In order to calculate criterion for fitness evaluation, the posteriori estimation error $\eta_{k, j}(i ; n)$ and criteria $J_{k, j}$, are defined as follows.

$$
\begin{aligned}
& \eta_{k, j}(i ; n)=\boldsymbol{\delta}^{T}(n) \mathbf{w}_{k, j}^{\mathbf{1}}(n+1)-d(i ; n)= \\
& e_{k, j}(i ; n)\left(1-\frac{\mu_{k, j}}{a+\boldsymbol{\delta}^{T}(n) \boldsymbol{\delta}(n)} \boldsymbol{\delta}^{T}(n) \boldsymbol{\delta}(n)\right)=e_{k, j}(n) \mathcal{Y}\left(\mu_{k, j}\right) \quad, 1 \leq j \leq R \\
& J_{k, j}=\sum_{n=1}^{T}\left[(1-\mathrm{P}) e_{k, j}(i ; n)^{2}+\mathrm{P} \eta_{k, j}(i ; n)^{2}\right] / T=(1-\mathrm{P}) J_{e_{k, j}}+\mathrm{P} J_{\eta_{k, l}}
\end{aligned}
$$

where $P$ is a parameter among $[0,1]$, and $J_{e_{k, j}}$, and $J_{n_{k, j}}$ espectively denote estimations of the meansquared priori and posteriori error in this evolution period.

As $n$ approaches infinity, the estimation of the excess mean-squared priori error of $J_{e_{k, j}}$, [2] can be approximated to

$$
J_{e_{k, j} ; \min } \sum_{i=1}^{M} \frac{\mu \lambda_{i}}{2-\mu \lambda_{i}}=J_{e_{k, j} ; \min } v(\mu)
$$

where $J_{e_{k, j}} ; \min$ is the minimum mean-squared priori error and $\lambda_{i}, 1 \leq i \leq M$ is the eigenvalues of correlation matrix of functional expansion units.

It is clear that $J_{e_{k j}}$, is a monotonic decreasing function of $\mu$ because $\nu(\mu)$ is a monotonic decreasing function of $\mu$. From equation (13), it is straight forward that $J_{n_{k, j}}$ becomes smaller than $J_{e_{k},}$, when $\mu$ increases due to that $\vartheta(\mu)$ is a monotonic decreasing function of $\mu$. From above discussion for $J_{e_{k, j}}$, and $J_{n_{k, j}}$ we find that the criteria $J_{k, j}$, with a larger $\mu$ will become smaller than that with a smaller $\mu$ when $P$ is large. Therefore, we let $P$ depend on the estimation of normalized mean-squared priori error. By this principle, a larger $P$ will be set to prefer a larger step-size to improve convergence rate when the priori error increases. The basic principle for adjusting parameter $P$ is expressed by

$$
\begin{gathered}
\mathrm{P}=\frac{J_{e_{k, 0}}}{J_{e_{k, \mathrm{max}}}} \\
J_{e_{k+1, \max }}=\gamma J_{e_{k, \max }}+(1-\gamma) J_{e_{k, 0}} \frac{v\left(\mu_{k, \max }\right)}{v\left(\mu_{k, 0}\right)},
\end{gathered}
$$

where $\gamma, 0<\gamma<1$, is a forget factor. Here, $J_{e_{k, 0}}$ and $J_{e_{k, \max }}$ respectively are estimates of mean-squared priori error corresponding to $\mu_{k, 0}$ and the maximum step-size in the kth evolution period. Finally, the optimal step-size $\mu_{k, o p t}$ which provides the minimum criteria in the $k$ th evolution period will be kept and be selected as the optimal parent step-size for next interval. Furthermore, its corresponding weight vector and filtered outputs are also kept.

\section{RESULT AND DISCUSSION}

\subsection{Simulations}

In order to estimate the efficiency of the proposed method for extracting evoked potentials, several simulations were carried out. The simulated trial sets with different SNR are first generated. A pattern of visual evoked potentials shown in Fig. 4 (a) was superimposed on realistic EEG multiplied by different gain to generate these trial sets.

Fig. 4 (b) shows four randomly selected trials with a SNR of $-10 \mathrm{~dB}$. Here, each trial set contains 1000 trials and each trial has 200 points. The SNR in this study is defined by

$$
S N R=20 \log _{10}\left(\frac{A_{s}}{A_{n}}\right) .
$$

$A_{s}$ and $A_{n}$ respectively denote the magnitudes of the signal of interest and noise.

Fig. 5 shows results obtained by using ALE, FLN, and FLN with GA algorithm for different step-sizes with SNR equal -10 dB. The adaptive line enhancement is a 32-tap filter. The parameters of FLN are set as follows: $N_{0}=16, N_{1}=32, \beta=0.1$. The number $R$ of offspring of step-sizes in GA algorithm is set to 7 . It clearly shows that the results obtained by ALE present heavy distortion. The result of FLN with $\mu=0.5$ fits the pattern of interest, but that with other step-sizes present distortions. The results of FLN using GA algorithm can always effectively track evoked potentials. Fig. 6 shows the error curve of each method with SNR equal $-10 \mathrm{~dB}$. In this simulation, the step-sizes of all above methods were set to 0.5 . The error curves of all above method approach the steady state after about 20 trials. The error curve of ALE is unstable.

In order to analyze the influence of the selection of step-sizes in different noise levels, the means of mean square error of each method obtained by averaging last 50 trials are investigated. Fig. 7 (a), Fig. 7 (b) and Fig. 7 (c) are respectively the means of mean square errors of each method under the SNR of $-5 \mathrm{~dB}$, $10 \mathrm{~dB}$ and $-20 \mathrm{~dB}$. It is clear that the performance of FLN is better than that of ALE. The FLN with GA algorithm always provides the optimum performance of FLN, and is insensitive to the selection of step-sizes. 


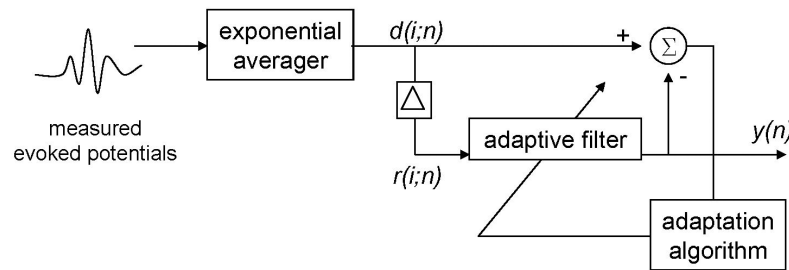

Fig. 1 Application of adaptive line enhancement for extracting evoked potentials.

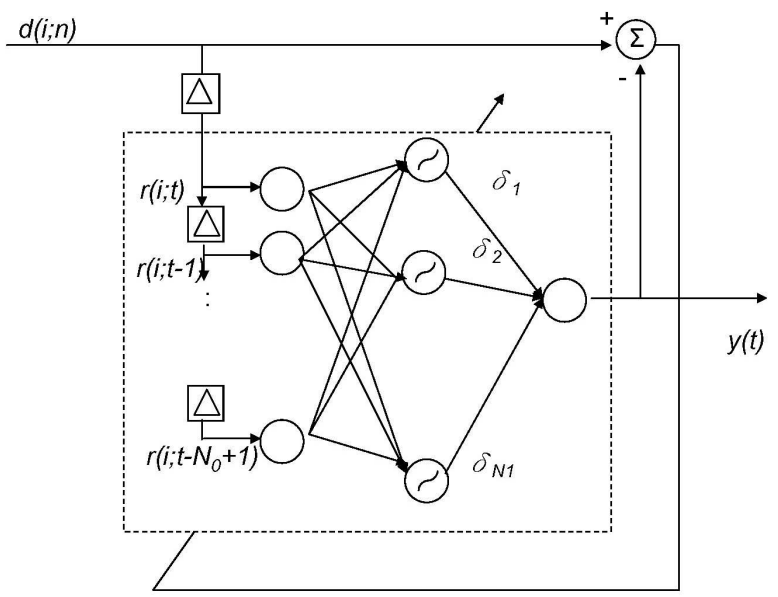

Fig. 2 Scheme of functional link network.

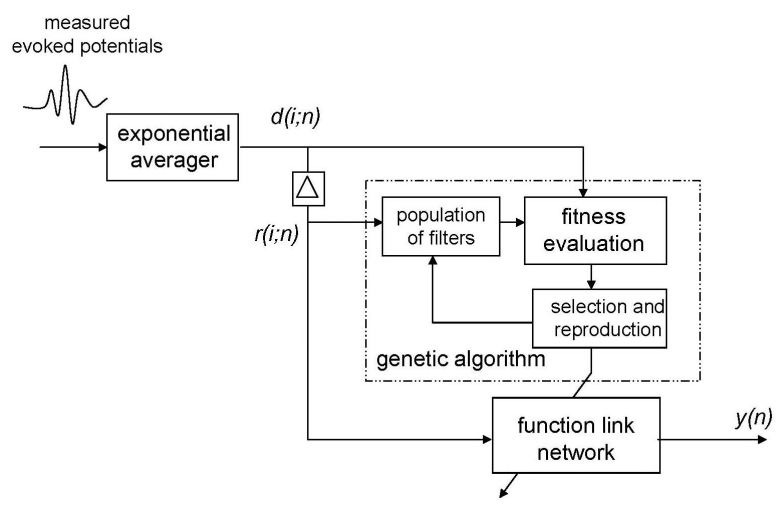

Fig. 3 Block diagram of functional link networks with genetic algorithm.

Both FLN and ALE with a smaller step-size can provide better performance when the SNR becomes poor. This can be explained from equation (8). The adaptation formula for weights is directly influenced by the error between the desired signal and filtered output, the selection of step-size, and the reference signals. When the SNR becomes poorer, variation of

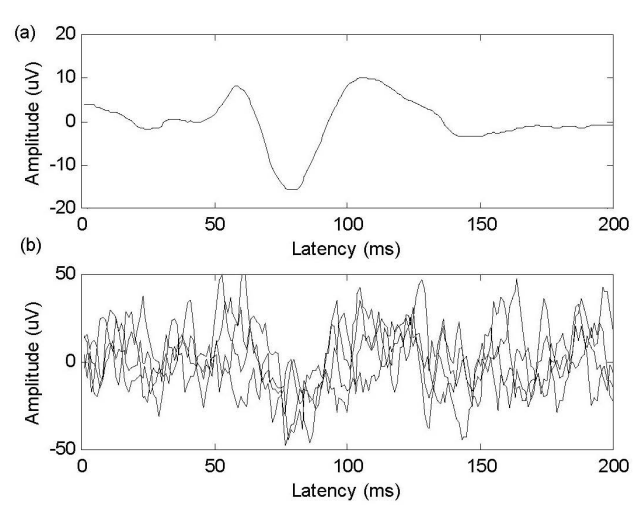

Fig. 4 (a) A pattern of evoked potentials for simulation (b) Four randomly selected noisy trials in the SNR of $-10 \mathrm{~dB}$.

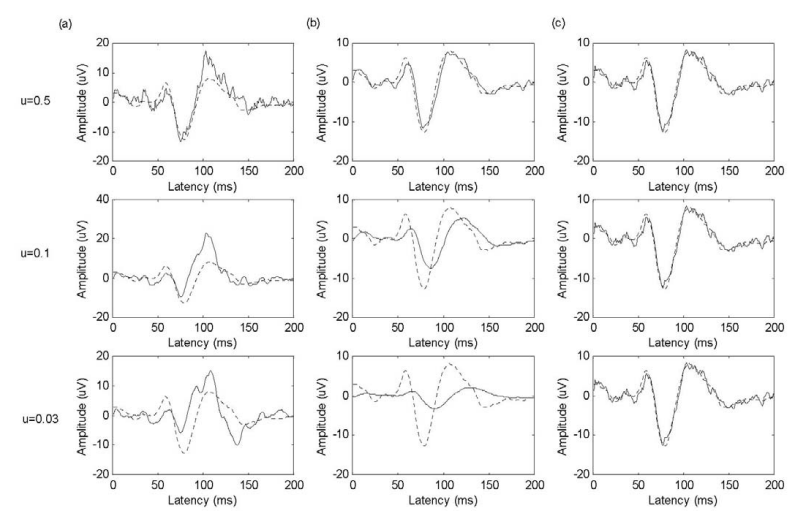

Fig. 5 Results of (a) adaptive line enhancement (b) functional link network (c) functional link network with genetic algorithm corresponding to step-sizes of $0.5,0.1$, and 0.03 under the SNR of $-10 \mathrm{~dB}$.

the error between desired signals and filtered output, and reference signals will become acute. Therefore, the steady state performance of each method with a large step-size under a poor SNR will present more unstable.

Fig. 8 shows the optimum performance of each method in different noise levels. It clearly shows that the optimum performance of FLN significantly is better than that of ALE. The FLN with GA algorithm always can provide the optimum performance of FLN in different noise levels and different step-sizes.

\subsection{Experiment}

Next, FLN with GA algorithm is applied to extract visual evoked potentials for a real human subject. A black and white checkerboard was used to evoke visual evoked potentials. Its cycle of visual stimulus is set to 1 second. This visual evoked potential response was 
sampled at $500 \mathrm{~Hz}$. A total of 1000 trials of visual evoked potentials were recorded. Each trial of the measured data set contains 500 points. This subject was drawn from a male subject of 23 years of age. The parameters of FLN with GA algorithm were set to $N_{0}=16, N_{1}=32, \beta=0.1$, and $M=7$. Fig 9. (a), Fig. 9 (b) and Fig. 9 (c) are respectively the randomly selected three results obtained by FLN with GA algorithm corresponding to different step-sizes of 0.5 , 0.1 and 0.03 . The solid line and the dotted line in the Fig. 9 respectively denote the filtered result of the proposed method and the ensemble average of 1000 real evoked potentials trials. It shows that the results of FLN with GA algorithm using different step-sizes are similar.

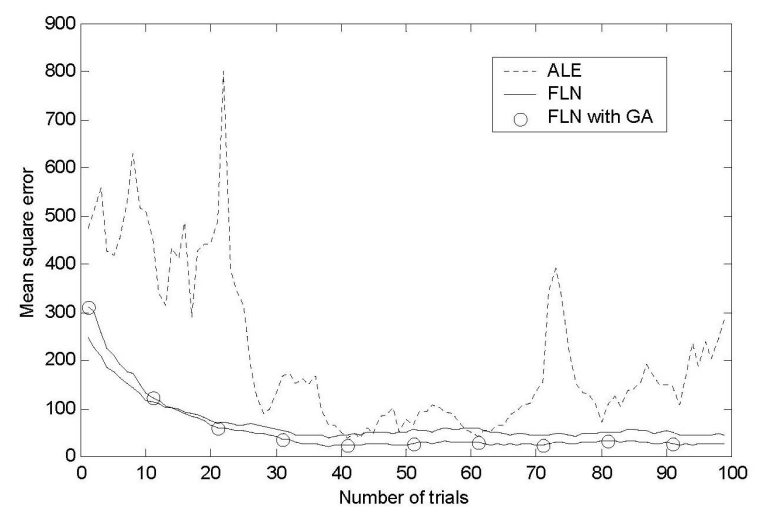

Fig. 6 Error curve of each method in the SNR of -10 dB.
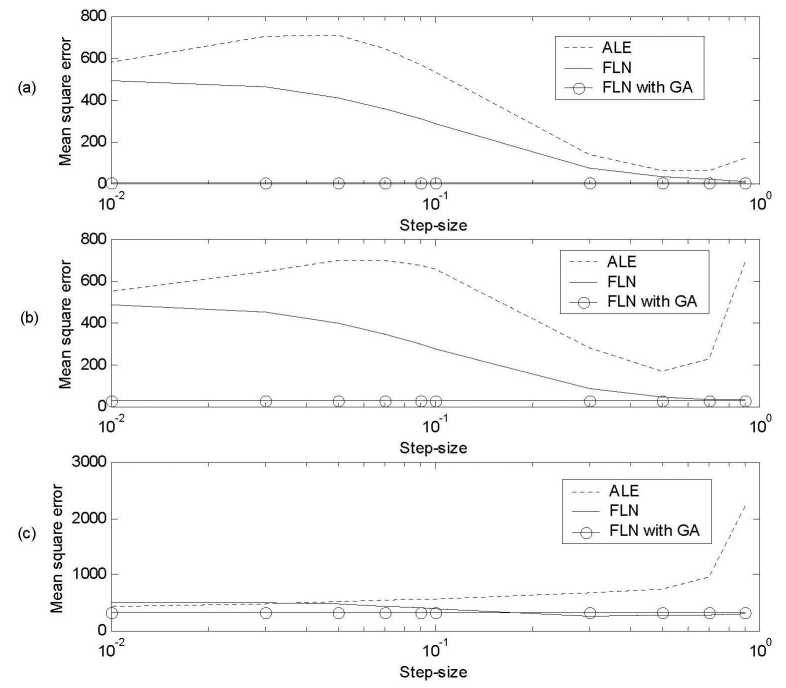

Fig. 7 Performance of each method corresponding to different step-sizes in the SNR of (a) -5 dB (b) -10 dB and (c) $-20 \mathrm{~dB}$.
Fig. 10 (b) and Fig. 10 (c) are respectively the variations of latencies and amplitudes of N1, P1, and N2 shown in Fig. 10 (a). Here, Fig. 10 (a) is the evoked potentials obtained by averaging 1000 real trials. The estimation of latencies and amplitudes is based on the fitting of mean square error between estimated evoked potentials and the vicinities of peaks of the evoked potential pattern. The width of the fitting window is set to 20 points. The standard deviation of estimated latencies and amplitudes of N1, P1, and N2 obtained by FLN using GA algorithm with different step-sizes are listed in TABLE I. It clearly shows that the track of the latencies of P1 and N2 is more stable than that of N1. The variations of the amplitudes of $\mathrm{N} 1, \mathrm{P} 1$, and $\mathrm{N} 2$ are similar. The variation of estimated latencies and amplitudes obtained by FLN with GA algorithm is insensitive to the selection of step-sizes. The variations of both latencies and amplitudes of N1, P1 and N2 present stable. Results show that the proposed method can effectively estimate the real evoked potentials.

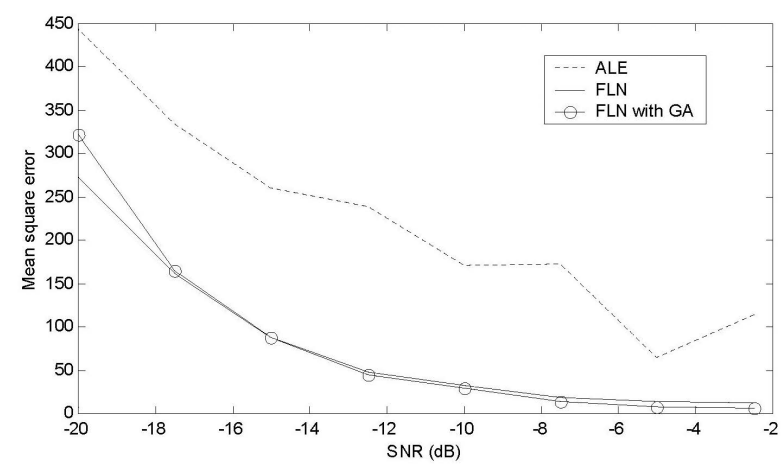

Fig. 8 The optimum performance of each method in different noise levels.
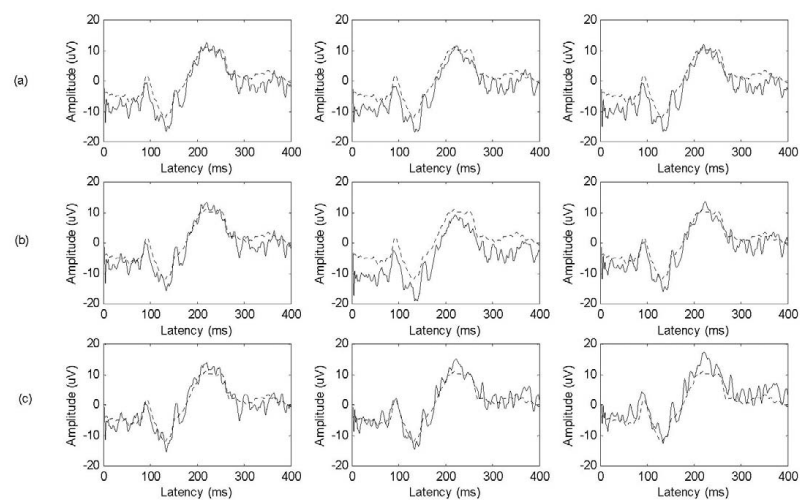

Fig. 9 Three randomly selected results of real evoked potentials by using step-sizes of (a) 0.5 (b) 0.1 (c) 0.03 . 


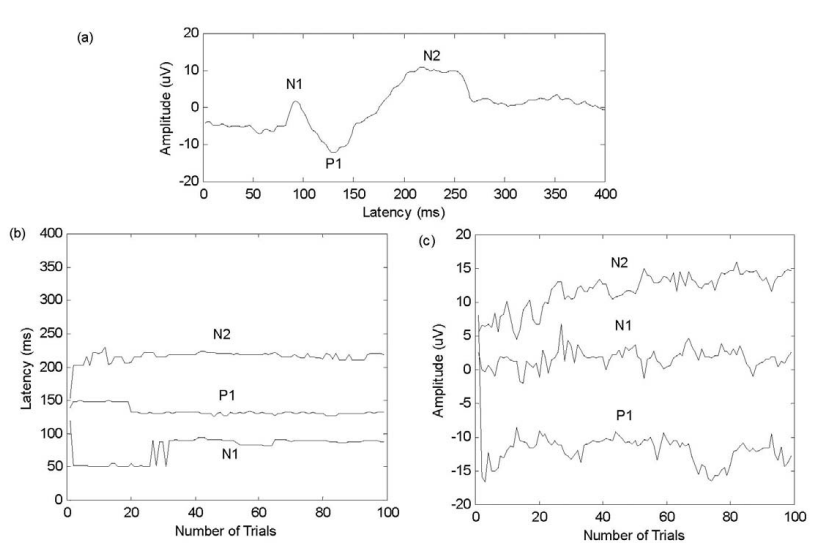

Fig. 10 (a) Ensemble average of 1000 trials of real evoked potentials (b) the variation of estimated latencies (c) the variation of estimated amplitudes obtained by FLN with GA.

\section{TABLE I}

\begin{tabular}{cccc}
\hline Step-size & $\begin{array}{c}\text { Peaks of } \\
\text { EP }\end{array}$ & $\begin{array}{c}\text { Standard deviation } \\
\text { of estimated } \\
\text { latency }\end{array}$ & $\begin{array}{c}\text { Standard deviation } \\
\text { of estimated } \\
\text { amplitude }\end{array}$ \\
\hline$\mu=0.5$ & $\mathrm{~N} 1$ & 17.549 & 1.3464 \\
\cline { 2 - 4 } & $\mathrm{P} 1$ & 6.9271 & 2.6216 \\
\cline { 2 - 4 } & $\mathrm{N} 2$ & 8.2389 & 2.6141 \\
\hline$\mu=0.1$ & $\mathrm{~N} 1$ & 17.4617 & 1.38 \\
\cline { 2 - 4 } & $\mathrm{P} 1$ & 6.9213 & 2.7089 \\
\cline { 2 - 4 } & $\mathrm{N} 2$ & 8.27 & 2.657 \\
\hline$\mu=0.03$ & $\mathrm{~N} 1$ & 17.4406 & 1.6069 \\
\cline { 2 - 4 } & $\mathrm{P} 1$ & 6.9889 & 2.6618 \\
\cline { 2 - 4 } & $\mathrm{N} 2$ & 8.4169 & 2.6542 \\
\hline
\end{tabular}

\section{CONCLUSION}

In this study, a genetic algorithm has been employed for extracting evoked potentials. By using regular generation and evolution of step-sizes, the step-size can be rapidly adjusted to optimum. It is useful for extracting evoked potentials under different types of evoked potentials and measured environments in the clinic. From simulations, the functional link network provides superior performance over that of the conventional adaptive line enhancement. However, its performance is also directly influenced by step-sizes. Simulated results show that employing genetic algorithm on FLN can always provides the optimum performance of FLN in different noise levels. Finally, from experiments of realistic human subject, it shows the efficient of extracting visual evoked potentials. Therefore, genetic algorithm can easily solve the issue of step-sizes. It is applicable for extracting evoked potentials in the clinic.

\section{REFERENCE}

1. C. D. McGillem, J. I. Aunon and D. G. Childers: Signal processing in evoked potential research: Application of flitering and pattern recognition. Critical Reviews in Bioengineering 1981; 9: 225265.

2. S. Haykin: Adaptive Filter Theory, 2nd. Englewood Cliffs, NJ: Prentice-Hall 1991.

3. N. V. Thakor: Adaptive filtering of evoked potentials. IEEE Transaction on Biomedical Engineering 1987; BME-34: 6-12.

4. C. A. Vaz and N. V. Thakor: Adaptive Fourier estimation of time-varying evoked potentials. IEEE Transactions on Biomedical Engineering 1989; 36: 448-455.

5. P. Laguna, R. Jane, O. Meste, P. W. Poon, P. Caminal, H. Rix, and N. V. Thakor: Adaptive for event-related bioelectric signals using an impulse correlated reference input: Comparison with signal averaging techniques. IEEE Transaction on Biomedical Engineering 1992; 39: 1032-1044.

6. P. G. Madhavan: Minimal Repetition Evoked Potentials by Modified Adaptive Line Enhancement. IEEE Transactions on Biomedical Engineering 1992; 39: 760-764.

7. O. Svensson: Tracking of changes in latency and amplitude of the evoked potential by using adaptive LMS filters and exponential averagers. IEEE Transaction on Biomedical Engineering 1993; 40: 1074-1079.

8. V. Parsa, and P. A. Parker: Multireference Adaptive Noise Cancellation Applied to Somatosensory Evoked potentials. IEEE Transactions on Biomedical Engineering 1994; 41: 792-800.

9. X. H. Yu, Z.Y. He, and Y-S. Zhang: Time-Varying Adaptive Filters for Evoked Potential Estimation. IEEE Transactions on Biomedical Engineering 1994; 41: 1062-1071.

10. R. Grieve, P. A. Parker, B. Hudgins, and K. Englehart: Nonlinear Adaptive Filtering of Stimulus Artifact. IEEE Transactions on Biomedical Engineering 2000; 47: 389-395.

11. S.H. Leung, C.Y. Chung, and S.C. Ng: A New Variable Step-size Algorithm using Genetic-type Search. Proceedings of the 1994 IEEE International Symposium on Circuits and Systems 1994; 2: 381384.

12. S.C. Ng, S.H. Leung, C.Y. Chung, A. Luk, and W.H. Lau: The genetic search approach: A new learning algorithm for adaptive IIR filtering. IEEE Signal Processing Magazine 1996; 13: 38-46. 
13. S.C. Ng, C.Y. Chung, S.H. Leung, and A. Luk: A variable step size algorithm using evolution strategies for adaptive filtering. Proceedings of the 1999 Congress on Evolutionary Computation 1999; 1: $542-545$.

14. W. K. Lennon, and K. M. Passino: Strategies for genetic adaptive control. Proceeding of the 36th conference on Decision and Control 1997; 19081913.
15. W. K. Lennon and K. M. Passino: Intelligent control for brake systems. IEEE Transactions on control systems technology 1999; 7: 188-202.

16. Y. H. Pao: Adaptive Pattern Recognition and Neural Networks. Addison-Wesley Publishing Company 1989.

17. N. E. Cotter: The Stone-Weierstrass Theorem and Its Application to Neural Networks. IEEE Transaction on Neural Networks 1990; 1: 290-295. 